\title{
IMPORTANCIA ECONÓMICA DE LOS ALEMANES EN VALPARAÍSO, 1850-1915*
}

\author{
ECONOMIC IMPORTANCE OF GERMANS \\ IN VALPARAISO, 1850-1915
}

\author{
Baldomero Estrada Turra \\ Pontificia Universidad Católica de Valparaíso, Valparaíso, Chile, <bestrada@ucv.cl>
}

\begin{abstract}
Resumen. El presente artículo se refiere al impacto económico de los alemanes en Valparaíso (1850-1915) y su relación con la expansión económica alemana. Se analiza la expansión de Alemania, como expresión del proceso de globalización, y sus repercusiones en Valparaíso. Esta presencia se observa tanto desde la acción de los inmigrantes como de capitalistas alemanes. Se estudia un ciclo evolutivo que termina con el inicio de la primera guerra mundial, que determinó la disminución de la colectividad y la decadencia económica de los comerciantes mayoristas. Paralelamente, se produce una notoria disminución de la actividad comercial de Valparaíso hacia fines del siglo XIX. Se concluye recalcando la relación que se establece entre las posibilidades de los colectivos inmigrantes y el potencial de sus países de origen en el contexto económico internacional.
\end{abstract}

Palabras clave: globalización, inmigración, comercio, mercado laboral, capitalismo.

Abstract. This article concerns the economic impact of Germans in Valparaiso (18501915) and their relationship with the German economic expansion. It analyzes the expansion of Germany, as an expression of the globalization process, and its impact in Valparaiso. This presence can be seen both from the action of German immigrants and German capitalists. We studied an evolutionary cycle that ends with the start of the First World War that determined the decline of the community, and the economic decline of the wholesalers. In parallel, a marked decrease is produced in commercial activity of Valparaiso toward the end of the nineteenth century. It is concluded by stressing the relationship that develops between the possibilities of the immigrant populations and the capability of their countries of origin in the international economic context.

Key words: globalization, immigration, trade, labor market, capitalism.

Fecha de recepción: marzo de 2012. Fecha de aceptación: junio de 2012.

* Comisión Nacional de Investigación Científica y Tecnológica, gobierno de Chile, Ministerio de Educación, Proyecto Fondo Nacional de Desarrollo Científico y Tecnológico, núm. 1100590. Agradezco a los evaluadores anónimos que contribuyeron al mejoramiento de este artículo.

Am. Lat. Hist. Econ., año 20, núm. 2, mayo-agosto, 2013, pp. 151-176 


\section{INTRODUCCIÓN}

$\mathrm{D}$ esde nuestra perspectiva, para el periodo estudiado, la inmigración es una de las dimensiones a través de las cuales se manifiesta el proceso de globalización. En virtud de ello es que consideramos en nuestro análisis el desplazamiento poblacional en forma separada a fin de hacer notar su relevancia. Efectivamente, el fenómeno de la globalización, comprendido fundamentalmente en el periodo de 1870 a 1914, se caracterizó por manifestarse en tres dimensiones: comercio de bienes y servicios, flujos de capitales y desplazamiento de personas. ${ }^{1} \mathrm{Y}$ aunque existe una relación íntima entre estos tres fenómenos, debemos considerar que no necesariamente son sincrónicos ni tampoco se comportan en los distintos lugares de manera similar. De allí nuestro interés en enfocar el proceso de globalización desde la inmigración de alemanes en Valparaíso sin perder de vista la estrecha vinculación que existe entre este desplazamiento humano y los vínculos comerciales y de capitales que se establecen también desde Alemania con el continente americano y en especial con Chile.

Aunque el momento de despegue de la ola de globalización surge hacia la década de 1870, coincidiendo con la unificación de Alemania, hemos preferido datar el inicio de este trabajo en 1850, sabiendo que los ciclos económicos se insertan en periodos cuyo desarrollo es paulatino y requiere de la conjunción de múltiples factores que se desarrollan y elaboran en etapas históricas de larga duración o, en el mejor de los casos, de mediana extensión. La presencia de Alemania en las costas americanas se manifestó desde Hamburgo y Bremen, una vez iniciada la independencia de Chile, expresando la relevancia que tenía el comercio internacional en dichos estados. La segunda mitad del siglo XIX testimonia, a escala mundial, los efectos de la revolución industrial y el reemplazo del mercantilismo por el liberalismo.

La actividad económica que realizan los colectivos inmigrantes alemanes en los diversos lugares del continente latinoamericano son, sin duda, un testimonio que expresa de muy buena forma el potencial que poseía Alemania dentro del contexto económico internacional. Los inmigrantes germanos actuaron como mediadores de un proceso económico, como también cultural, entre su sociedad de origen y la sociedad receptora.

En general, la bibliografía sobre inmigración alemana en América Latina se ha detenido preferentemente en los procesos de colonización, como ha ocurrido en la mayoría de las investigaciones realizadas para

\footnotetext{
${ }^{1}$ Parodi, “América”, 2007, p. 5.
} 
Brasil y Argentina. ${ }^{2}$ Para el caso de Chile tal situación es palmaria. ${ }^{3}$ Una investigación que apunta en nuestra perspectiva la encontramos para México, realizada por Brígida von Mentz, quien plantea, para fines del siglo XIX, que la inmigración germana en dicho país estuvo constituida por "representantes del capitalismo comercial e industrial alemán". ${ }^{4}$ Para el caso de Costa Rica, Eugenio Herrera Balharry se refiere a la importancia de los alemanes en el comercio internacional y su vínculo con la explotación cafetalera, principal producto de exportación costarricense, que los lleva a constituir parte importante de la elite local. ${ }^{5}$

Cabe consignar que la corriente migratoria alemana hacia los países latinoamericanos fue muy exigua, yéndose la mayor parte $(91 \%)$ de dicha inmigración hacia Estados Unidos de Norteamérica. ${ }^{6}$ De acuerdo con fuentes alemanas, para el periodo comprendido entre 1848 y 1914, los emigrantes que salieron con destino a Chile desde los estados alemanes fueron 13 945, lo que representaba $0.2 \%$ del total de 4500000 emigrantes alemanes que salieron durante dicho periodo. ${ }^{7}$ Los alemanes que llegaron a Brasil fueron alrededor de $250000^{8}$ y a Argentina $25000,{ }^{9}$ cantidades reducidas para estos países receptores frente a la masiva inmigración que recibieron desde otros países europeos.

\section{INMIGRACIÓN EUROPEA EN CHILE: UN PROCESO DE DESARROLLO EMPRESARIAL}

Como es sabido, el proceso migratorio europeo hacia América Latina, que inició a mediados del siglo XIX, se caracterizó por ser masivo y se orientó fundamentalmente a los países de la costa atlántica, especialmente hacia Brasil y Argentina. La fuerte oferta laboral impuesta por la explotación del café y del trigo y sus atractivos salarios orientaron a millones de inmigrantes hacia dichos países.

Chile no poseía una economía que requiriera gran cantidad de mano de obra, pero sí consideró necesario atraer a inmigrantes europeos a fin de ocupar territorios, contar con mano de obra especializada o simple-

${ }^{2}$ Seyferth, "Inmigración”, 1995, y Vera, "Emigraciones", 1994.

${ }^{3} \mathrm{Al}$ respecto véanse Young, Germans, 1974, y Blancpain, Allemands, 1974.

${ }^{4}$ Mentz, Pioneros, 1982, p. 6.

${ }^{5}$ Herrera, Alemanes, 1988.

${ }^{6}$ Vera, "Emigraciones", 1994, p. 82.

7 Ibid., pp. 80-82, y Kellenbenz y Schneider, "Emigración”, 1976, p. 402.

${ }^{8}$ Vera, "Emigraciones", 1994, p. 82.

${ }^{9}$ Seyferth, "Inmigración", 1995 , p. 55. Al igual que en la mayoría de los casos de América Latina hay discrepancias según el origen de la información y las cifras varían entre 230000 y 280000 inmigrantes. 
mente incrementar su población, que por razones económicas y de estrategia geopolítica se veía disminuida ante el extraordinario crecimiento de los vecinos de este país, especialmente Argentina. Para Benjamín Vicuña Mackenna, connotado político e intelectual de la segunda mitad del siglo XIX, la inmigración europea resolvía varios problemas a la vez, entre ellos el del territorio, ocupando zonas que podrían interesar a los vecinos chilenos; la carencia de técnicas modernas; el de la organización como nación y el problema de El Arauco, es decir, la expulsión y sometimiento de los indígenas que ocupaban dicha región. ${ }^{10}$ Se veía la venida de los inmigrantes europeos como un aporte material pero también cultural y moral, como explícitamente lo proclamaba Joaquín Villarino en 1867, orientado a estimular la inmigración: "Bienvenida sea la inmigración europea porque aporta consigo el adelanto moral para nuestras masas ignorantes; introduce entre nosotros prácticas útiles y contribuye a cimentar la paz y la prosperidad, el progreso en las instituciones y la libertad [...] Salud a esa inmigración que lleva consigo el estandarte de la igualdad, de la fraternidad y el progreso universales." 11

Los primeros intentos estatales, fruto de políticas específicas y orientadas a atraer inmigrantes europeos, se iniciaron a mediados del siglo XIX, cuando se trajeron algunas familias alemanas que radicaron en las provincias de Valdivia y Llanquihue. Después en la década de 1980 se llevaron a cabo acciones más decididas con la intención de atraer agricultores europeos. Diversas dificultades frustraron este proyecto y la mayoría de estos supuestos colonos terminaron instalándose en pequeñas ciudades de los alrededores. Posteriormente, se pretendió conseguir trabajadores urbanos que pudieran estimular el naciente parque industrial nacional, pero tampoco fue posible lograr tales objetivos ya que los cerca de 30000 inmigrantes que llegaron con tal objetivo entre 1880 y 1891 en su mayoría reemigraron a Argentina.

Dado lo anterior, la inmigración que finalmente se radicó en el país y consolidó la conformación de colectividades europeas que permanecen hasta hoy, se desarrolló al alero de redes urbanas constituidas fundamentalmente por comerciantes que en su desarrollo empresarial requirieron de colaboradores, lo que estimuló el funcionamiento de redes étnicas que nutrieron tales requerimientos. Las ciudades más importantes, como Santiago y Valparaíso, experimentaron un notorio proceso de modernización a fines del siglo XIX, generando importantes posibilidades de desarrollo para el quehacer comercial e industrial que fueron rápidamente aprovechadas por inmigrantes europeos.

${ }^{10}$ Vicuña, Bases, 1865, pp. 10-14.

${ }^{11}$ Villarino, Estudios, 1867, p. 171. 
Otra vía que posibilitó la llegada de extranjeros fue el arribo de comerciantes mayoristas que incorporaron a Valparaíso al circuito comercial internacional del Pacífico Sur. Es justamente la modalidad migratoria que nos interesa analizar en esta investigación. Se trata de aquellos funcionarios de sucursales de casas establecidas en Europa y que operaban a través de las costas americanas como importadoras de productos naturales o exportadoras de artículos europeos de fácil colocación en los mercados americanos. Relacionadas a esta actividad comercial internacional, propia del proceso expansivo de globalización de fines del siglo XIX, encontramos también a otras instituciones como los bancos, representantes de compañías navieras y compañías de seguros que propiciaban el traslado de funcionarios europeos que debían permanecer en las distintas filiales repartidas por el mundo trabajando por periodos limitados y procurando no establecer mayores vínculos con la sociedad nativa. Este grupo de alemanes distribuidos alrededor del mundo son los denominados Reichsdeutsche. ${ }^{12}$ Este tipo de inmigrante lo encontramos fundamentalmente en las colectividades británicas y alemanas, y se caracterizaban por desarrollar la mayor parte de sus actividades comerciales y sociales al interior de su comunidad o con otros extranjeros vinculados al comercio internacional y, excepcionalmente, con miembros de la elite local. Eran muy comunes los vínculos entre alemanes y británicos, donde eran habituales las sociedades en donde coparticipaban miembros de ambos colectivos.

Este grupo de comerciantes concentrados en casas importadoras e instituciones financieras, dependientes de casas matrices establecidas en Alemania, constituyeron un grupo dentro de la colectividad inmigrante con características especiales. Se trata de inmigrantes temporales por cuanto habitualmente vienen por periodos definidos como funcionarios. También encontramos, en las sociedades constituidas como casas importadorasexportadoras, socios que nunca vinieron a Chile. ${ }^{13}$ Por otro lado, existe también el caso de familias, algunas de cuyas ramas finalmente se establecieron en Chile, como ocurrió con la familia Claude.

La presencia europea en Valparaíso, por consiguiente, se explica particularmente por su posición portuaria y su importante participación en el quehacer comercial controlado por Europa, especialmente por británicos y alemanes. El caso concreto del principal puerto de Valparaíso es muy elocuente por la sobresaliente concentración que tuvo de europeos que lideraron la actividad económica, superando a los propios nativos y mos-

${ }^{12}$ Young, Germans, 1974, p. 13.

${ }^{13}$ Couyoumdjiam, "Alto", 2000, p. 69, refiere que la casa comercial Vorwerk tenía entre sus socios a seis miembros de la familia Vorwerk que residían en Alemania mientras en Chile dirigía la casa Juan Carlos Fisher, hijo de Hermann Fisher, anterior gerente, quien dirigió la empresa hasta 1903, meses antes de fallecer luego de residir más de 50 años en Chile. 
trando una extraordinaria sobrerrepresentación en el sector productivo en relación con su menguada presencia demográfica. Mary Graham, a comienzos de la década de 1820, hacía notar el predominio de mercaderías extranjeras en las tiendas de la ciudad, en donde se podía encontrar "sedas de China, Francia e Italia, telas de algodón de Gran Bretaña; rosarios, amuletos y cristales de Alemania". Agregaba que raramente se podían adquirir artículos nacionales, ya que su producción era fundamentalmente para el consumo familiar doméstico. En cuanto a los alemanes, precisaba que eran quienes proveían la cristalería de uso corriente, que eran de mala calidad pero respondían a las necesidades de la población. También ofrecían peines, juguetes, perfumes y artículos religiosos. Hace especial mención de la presencia de un herrero y veterinario alemán de apellido Frey cuyo taller y casa llamaban la atención por su hermosura y aseo. ${ }^{14}$

La evolución de la población europea es creciente a través del siglo XIX, aumentando su participación demográfica como también su mayor injerencia en la economía local. De acuerdo con el censo de 1907, cuando logran un mayor porcentaje en representación, los europeos existentes en Valparaíso representaban poco más de 7\% de la población pero poseían $50 \%$ del control de la actividad económica de la ciudad, sobre todo en los negocios y actividades de mayor capitalización en el ámbito comercial y de mayor tecnología en el plano industrial. 1907 es también el año en que la colectividad alemana alcanza la cifra más alta para el periodo en estudio (véase cuadro 1).

Cabe precisar que el grupo de europeos establecidos en Valparaíso, desde mediados del siglo XIX, estaba constituido fundamentalmente por británicos, alemanes, españoles, italianos y franceses. La distribución numérica y su participación en la economía de la ciudad se vinculaban directamente con el posicionamiento que tenían sus respectivos países dentro del concierto económico internacional. Es así como los británicos y alemanes controlaban el comercio mayorista como también las industrias más desarrolladas. Luego se ubicaban los franceses y finalmente los españoles e italianos, que operaban fundamentalmente en el comercio minorista. La presencia de los europeos del norte, a través del tiempo, mantuvo una estrecha relación con la relevancia que tuvieron en el plano internacional, por lo que luego de la primera guerra mundial experimentaron una disminución significativa. Por el contrario, los inmigrantes procedentes del sur europeo, que venían buscando mejores destinos, escapando de los apremios que afectaban a sus países, fueron aumentando su presencia y fortaleciendo su estructura económica como colectividades que, por lo demás, se desarrollaban acorde a los esfuerzos desplegados sólo por ellos, con capi-

${ }^{14}$ Graham, Diario, 1988, pp. 23-24. 


\section{CUADRO 1. COLECTIVIDADES EUROPEAS MÁS IMPORTANTES \\ EN EL DEPARTAMENTO DE VALPARAÍSO, 1865-1930 \\ (EN PORCENTAJES)}

\begin{tabular}{lrrrrrrr}
\hline Nacionalidad & 1865 & 1875 & 1885 & 1895 & 1907 & 1920 & 1930 \\
& & & & & & & \\
Alemanes & 15.68 & 16.38 & 15.20 & 13.55 & 14.04 & 10.71 & 11.56 \\
Franceses & 16.00 & 14.24 & 10.68 & 10.65 & 6.85 & 5.88 & 4.22 \\
Españoles & 8.87 & 4.99 & 7.33 & 12.78 & 23.67 & 26.00 & 23.39 \\
Británicos & 20.31 & 25.79 & 19.29 & 19.16 & 14.03 & 13.38 & 10.17 \\
Italianos & 9.49 & 11.66 & 18.91 & 21.98 & 20.40 & 21.10 & 21.80 \\
Otros extranjeros & 29.62 & 26.21 & 28.56 & 21.92 & 20.99 & 22.91 & 28.83 \\
Total en números & & & & & & & \\
$\quad$ absolutos & 4992 & 6921 & 7662 & 10300 & 14630 & 13443 & 12995 \\
\hline
\end{tabular}
1930.

Fuentes: elaboración propia con base en Censos, años 1865, 1875, 1885, 1895, 1907, 1920 y

tales obtenidos con su trabajo y sin mayores ventajas u aportes prodigados desde sus países y, por el contrario, eran ellos quienes habitualmente enviaban remesas para ayudar a los familiares que quedaron en casa.

De allí entonces que el proceso inmigratorio en Valparaíso sólo se explica a partir de las posibilidades que aparecen para quienes están dispuestos a incursionar en actividades de autoempleo o de desarrollo empresarial, explotando las redes migratorias, las posibilidades que surgen del posicionamiento de sus países de origen, sus particulares capacidades culturales como las facilidades que les otorga el medio que los acoge.

\section{PRESENCIA ALEMANA Y COMERCiO INTERNACIONAL}

Tal como lo afirmamos, muchos de los alemanes, como también ocurría con los británicos, llegaron a Valparaíso a desempeñarse como funcionarios de empresas o instituciones cuyas casas matrices se encontraban en Alemania y su gestión en el principal puerto de Chile era temporal, sabiendo que luego de un periodo determinado, habitualmente de tres años, eran destinados a otro lugar. La firma Huth Gruning, establecida en Londres y con sucursales en Lima y Valparaíso, tenía prohibido a los socios establecidos en dichas ciudades contraer matrimonio con mujeres 
peruanas o chilenas sin el consentimiento de los socios mayoritarios. ${ }^{15} \mathrm{Se}$ evitaba así mayor involucramiento con la sociedad nativa, garantizando la independencia operativa de la casa comercial. ${ }^{16}$

Existía, en todo caso, otro grupo de inmigrantes alemanes que no poseía estos vínculos de dependencia laboral y no estaba sometido a las limitaciones de vínculos personales que tal condición les imponía, como ocurría en el caso de las limitaciones matrimoniales a que estaban sujetos los socios de Huth Gruning.

El limitado mercado laboral porteño y sus bajos salarios hacían muy poco atrayente para los inmigrantes insertarse en el mercado urbano existente en Valparaíso. Sin embargo, las amplias posibilidades emergentes que surgían del comercio local, de poca atracción para la población nativa, constituyeron un nicho laboral muy atractivo para los foráneos. Es evidente que el comercio internacional, controlado por extranjeros, posibilitó mejores expectativas para que también fueran europeos quienes se asumieran como representantes y distribuidores de productos importados, lo que generó una red que comprometió todo el ciclo comercial, desde la importación hasta el expendio al consumidor. Los inmigrantes alemanes que no llegaban vinculados a empresas o instituciones que representaban a casas matrices germanas, se desempeñaban también en su mayoría en actividades comerciales o industriales que muchas veces usufructuaban los beneficios de contar con casas importadoras, bancos, aseguradoras y líneas de navegación alemanas, lo cual facilitaba su quehacer y les daba importantes ventajas en su gestión.

\section{Casas importadoras}

La evolución que tuvo la colectividad alemana, luego de lograda la independencia en Chile, fue lenta y, para el caso de Valparaíso, fue desarrollándose, en el transcurso del siglo XIX, en la medida del incremento de la importancia económica que fue adquiriendo dicho puerto. Los primeros antecedentes surgen a comienzos de la década de 1820 con el establecimiento de las primeras casas comerciales y la llegada de navíos desde Hamburgo y Bremen. La primera casa comercial que se estableció fue Schutte, Post y Cía., en 1822. Posteriormente, en 1824, se instalaron Huth (más tarde Huth Gruning y Cía.) y Johannes Stuven. ${ }^{17}$

\footnotetext{
${ }^{15}$ Cavieres, Comercio, 1988, p. 168.

${ }^{16}$ También existían limitaciones similares para los británicos.

${ }^{17}$ Kellenbenz, "Eduard", 1987, p. 26, y "Relaciones", 1988, pp. 120-121.
} 
El incremento de la presencia de comerciantes alemanes trajo consigo la necesidad de tener representantes diplomáticos por parte de las diferentes ciudades y repúblicas hanseáticas. Dado que se trataba de cargos ad honorem y su principal función era facilitar las actividades comerciales, quienes desempeñaron estos cargos fueron sobresalientes comerciantes establecidos en la ciudad. Los primeros nombramientos se efectuaron en 1835, cuando se elige a Simón von Post como cónsul de la república y ciudad libre y hanseática de Bremen, y a August Hermann Kindermann como cónsul de Hamburgo. Von Post era uno de los socios de la mencionada casa Schutte, Post y Cía., que pertenecía a una familia de fuertes lazos comerciales y familiares con importantes familias de Bremen instaladas en Estados Unidos de Norteamérica, Sudamérica y Bremen, como los Meier, Delius y Gildemeister. Por su parte, Kindermann era cajero en la casa Huth Gruning y Cía., quien tuvo una activa participación como cónsul en la venida de colonos alemanes a Chile. Después, ocuparon los cargos consulares de ambas representaciones otros comerciantes con experiencias similares. A von Post lo sucedió su socio William S. Leese, y a Kindermann lo reemplazó Ferdinand Mutzebecher, que venía de Lima, en donde había ocupado el mismo cargo diplomático. ${ }^{18}$

Entonces, otros estados alemanes fueron nombrando también a sus representantes. En 1838 lo hicieron Frankfurt, que designó a Felipe Bayerbach; Meckenburg a R. Behrens, Hannover a Federico Diestel, Sachsen a D. O. Richter, y Lübeck a F. Krock. En 1848, Preussen designó a Eduardo Müller; en 1866, Oldemburg nombró a Arnoldo Prost. Finalmente, en 1867, asumió Carlos Pini como cónsul general de la Federación de Estados del Norte. ${ }^{19}$

A mediados del siglo XIX la actividad comercial del principal puerto de Chile recibió el fuerte impulso del descubrimiento de oro en California. Valparaíso fue paso obligado de los cientos de navíos que se dirigían a ese explosivo centro minero que también fue un muy buen mercado para el trigo chileno, al menos durante la década de 1850. Al observar las fuentes de dicho periodo podemos notar cómo este desarrollo comercial estuvo acompañado por un aumento demográfico y económico de la colectividad alemana en la ciudad-puerto. En el cuadro 2 podemos advertir el crecimiento de las casas importadoras, en general, y en particular de los alemanes, y el impulso que tuvo dicha actividad para entonces. El fuerte predominio extranjero, especialmente europeo, es elocuente, como también lo es el significativo aumento que tienen las casas mayoristas de comercio. Cabe señalar que del listado de casas de consignación de 1857 tan sólo tres aparecían en la nómina de 1847; estas eran D. Shutte y Cía.,

${ }^{18}$ Kellenbenz, "Relaciones", 1988.

${ }^{19}$ Viebrock, "Historia", 1997, p. 32. 


\section{CUADRO 2. CASAS DE CONSIGNACIÓN EN VALPARAÍSO SEGÚN NACIONALIDAD, 1847-1895 (EN PORCENTAJES)}

\begin{tabular}{lcccrr}
\hline Nacionalidad & 1847 & 1857 & 1875 & 1885 & 1895 \\
Chilenas & 21.05 & 27.64 & 10.52 & 15.44 & 20.15 \\
Británicas & 28.07 & 24.39 & 26.31 & 23.57 & 12.40 \\
Alemanas & 19.29 & 15.44 & 26.31 & 30.08 & 29.45 \\
Francesas & 12.28 & 13.82 & 18.94 & 8.94 & 6.20 \\
Españolas & 5.26 & 8.13 & 3.15 & 3.25 & 10.07 \\
Estadunidenses & 8.77 & 3.25 & 3.15 & 2.43 & 2.32 \\
Otras & 5.26 & 7.31 & 11.57 & 16.26 & 19.37 \\
Total en números absolutos & 57 & 123 & 95 & 123 & 129 \\
& & & & & \\
\hline
\end{tabular}

Fuentes: para 1847 véase "Guía”, 1846; para 1857, Guía, 1858; para 1875, Chaigneau, Guía, 1874; para 1885, "Matrícula de patentes de Valparaíso para el año 1885", La Patria, 8 de junio de 1885, y para 1895, "Matrícula de patentes de Valparaíso para el año 1895", El Heraldo, 19 de junio de 1895 .

D. Rosemberg y Cía., y Huth Gruning. Las restantes 17 casas importadoras eran nuevas. Esto revela la permanente movilidad que tenía la mayoría de estos comerciantes y, por lo tanto, es una minoría la que se establece por periodos prolongados, como lo es el caso de las dos ya mencionadas casas, que se fundaron respectivamente en 1822 y 1828.

La disminución que muestra el número de casas importadoras, para la década de 1870, se relaciona directamente con la crisis que afectó a la economía chilena para entonces y que sólo se superó con la incorporación del salitre, luego de la guerra contra Perú y Bolivia. ${ }^{20}$ Posteriormente se produjo una notoria recuperación, que se mantuvo hasta el inicio de la primera guerra mundial. Es así como en 1913 el número de casas importadoras alemanas llegó a 43 establecimientos, pero para 1925 cayó a $25 .^{21}$

Los inicios de Huth Gruning se encuentran en las actividades comerciales llevadas a cabo por el alemán Frederick Huth desde la Coruña en 1805, desde donde comercializaba mercaderías inglesas, especialmente acero. Amplió luego su circuito a otras ciudades españolas para, después, instalarse en Londres, en 1808, con un reducido capital de 700 libras esterlinas. En 1819 adquirió la nacionalidad británica y junto al bremense F. Gruning fundó la casa comercial que tanto prestigio e importancia tuvo, especialmente

\footnotetext{
${ }^{20}$ Ortega, Chile, 2005.

${ }^{21}$ Pena, Guía, 1913, pp. 67-69, y Guía, 1926, pp. 674-676.
} 
durante la segunda mitad del siglo XIX, con un capital de 30000 libras esterlinas, del cual 75\% fue aportado por Huth (véase cuadro 3). ${ }^{22}$

La firma de Valparaíso se instaló con un capital de 200000 pesos chilenos, legalmente independiente de la casa matriz pero sujeta a diversas limitaciones impuestas por estas que la transformaban en agencia dedicada a transar mercaderías británicas y algunas francesas. Para 1840 las importaciones representaban 1000000 de pesos anuales. Entre las prohibiciones que tenían los socios establecidos en Perú y Chile estaba la de contraer matrimonio con nativas sin previo consentimiento de los socios mayoritarios. Tampoco podían conceder préstamos a los gobiernos locales.

Una vez instalados en Valparaíso, pronto se establecieron también en Santiago y en Tacna, que para entonces estaba en poder de las autoridades chilenas. En el norte fue muy importante el vínculo que establecieron con la actividad minera, a través de cuentas o garantizando créditos de la banca, como fue el caso de México South America Mining Company, que obtuvo recursos del Union Bank de Glasgow por vía de la participación de Huth Gruning. ${ }^{23}$

No hay duda que el movimiento que tuvo la casa fue sobresaliente para el periodo de 1850 a 1880. Las utilidades obtenidas eran el resultado de diversos pagos, como comisiones, intereses, garantías, etc. Cabe agregar que en estas cifras no se consideran ganancias obtenidas por inversiones de capital, como era el caso de compra de acciones de bancos en donde, en virtud de la importancia de su inversión, constituían parte del directorio como fue el caso del banco de Valparaíso, en donde de los seis directores, tres representaban a casas mayoristas extranjeras. Igualmente invirtieron en otras sociedades, como la Compañía Chilena de Seguros, la Compañía de Vapores y Remolcadores de Valparaíso, la Compañía de Diques Flotantes de Valparaíso y otras compañías mineras o comerciales. ${ }^{24}$ Las ganancias obtenidas por la casa de Valparaíso se distribuían dejando $50 \%$ para los socios de Valparaíso, quienes las recibían al dejar la ciudad o luego de cinco años. El resto se enviaba a Londres.

La importancia del desarrollo del principal puerto de Valparaíso como entrepôt se acentúa a mediados del siglo XIX, lo que se evidencia en la emergencia de nuevas casas de consignación que representan a comerciantes procedentes de diversos países. En el plazo de menos de diez años se duplican estos establecimientos, cuya función de importadores y exportadores se proyectaba más allá de las propias necesidades del país. Efectivamente, muchas de las mercaderías que llegaban a Valparaíso eran reembarcadas

\footnotetext{
${ }^{22}$ Cavieres, Comercio, 1988, p. 167.

${ }^{23}$ Ibid., p. 169.

${ }^{24}$ Ibid.
} 


\section{CUADRO 3. UTILIDADES NETAS DE HUTH GRUNING}

EN VALPARAÍSO, 1851-1880

\begin{tabular}{lrrrrr}
\hline Año & Utilidad & Año & Utilidad & Año & Utilidad \\
1851 & 163000 & 1861 & 90000 & 1871 & 120000 \\
1852 & 189000 & 1862 & 200000 & 1872 & 156000 \\
1853 & 125000 & 1863 & 138000 & 1873 & 117000 \\
1854 & 223000 & 1864 & 200000 & 1874 & 103000 \\
1855 & 268000 & 1865 & 80000 & 1875 & 28000 \\
1856 & 212000 & 1866 & 220000 & 1876 & 36000 \\
1857 & 140000 & 1867 & 160000 & 1877 & 56000 \\
1858 & 70000 & 1868 & 113000 & 1878 & 36000 \\
1859 & 188000 & 1869 & 107000 & 1879 & 307000 \\
1860 & 170000 & 1870 & 77000 & 1880 & 158000 \\
\hline
\end{tabular}

Fuente: documentos de la compañía Guldall Library, Huth Gruning \& Co., en Cavieres, Comercio, 1988, p. 171.

a otros puertos del Pacífico sur, como Ecuador, o los de Centroamérica, o abastecían la región noreste de Argentina o el sur de Perú y el sureste de Bolivia. ${ }^{25}$ Para las décadas de 1840 y 1850, los ingresos fiscales procedentes de aduanas constituían 60\% del total recibido por el Estado chileno. ${ }^{26}$ Se producía así una interdependencia americana y una dependencia de Europa, especialmente de Gran Bretaña. Tal situación estimulaba que la política económica fiscal fuese cuidadosa al establecer imposiciones al comercio internacional que debía, además, enfrentar efectos de conflictos con los vecinos de Chile, las revoluciones internas como también los problemas políticos europeos fueron parte del periodo revolucionario de 1848. Dicho año fue de malas consecuencias para el comercio de Valparaíso, que pudo levantarse con nuevos bríos gracias al gold rush californiano y luego el australiano.

Entre las casas importadoras mayoristas existentes durante la primera mitad del siglo XIX destaca Vorwerk y Cía., que se inició en Valparaíso en 1846 bajo la razón social de Otto Unde, Hüniken y Cía. Luego se transformó en Hüniken, Bahr \& Cía., Julius Hüniken era yerno de Georg Friedrich

\footnotetext{
${ }^{25}$ Garreau, "Formación”, 1984.

${ }^{26}$ Ibid., p. 183.
} 
Vorwerk, quien era socio, desde 1923 en Hamburgo, del almacén general y empresa de transporte Hochgreve \& Vorwerck. ${ }^{27}$

En 1860 llegó a hacerse cargo de la firma Adolph Vorwerk, quien luego de permanecer cerca de diez años en Chile regresó a Hamburgo. Durante los primeros años se ocupó especialmente del comercio salitrero. En los años 1880-1881 la firma tuvo la consignación de todo el salitre que se envió a Europa por cuenta del gobierno de Chile. Proveyó también al gobierno del fusil Mauser y otras armas de artillería. ${ }^{28}$

Para 1915 los socios de la empresa en Valparaíso eran John Fisher (hijo de Herman, quien estuvo a cargo de la gerencia de la empresa durante 25 años $)^{29}$ y Max Grisar. Se dedicaban a realizar operaciones bancarias, transacciones monetarias, exportación de minerales e importación de mercaderías en general. Representaban a la Compañía Naviera Kosmos y habían tomado parte en la creación de esta importante naviera. Representaban también a empresas de ferrocarriles como Antofagasta \& Bolivia Railway y Co. Ltd., y compañías de seguros como North British \& Mercantile Insurance Co. Operaban también como representantes de importantes fábricas alemanas, como Krupp (material ferroviario y minero), Deutsche Waften und Munition Fabriken, que instaló las máquinas de la fábrica de cartuchos de Santiago, y Henschel \& Sohn (locomotoras). También tenían parte en la propiedad de la fábrica de tejidos Victoria de Puente Alto, cuyo directorio, para 1915, estaba compuesto por John Fisher (presidente), J. E. Pini, Carlos Aldunate, M. J. Grisar, y Alfred Muller (gerente). Las oficinas principales de esta industria, que tenía más de 400 operarias, estaban ubicadas en Valparaíso. ${ }^{30}$

Para 1930, cuando su capital era de 500000 dólares estadunidenses, se mantenían como socios comanditarios Fisher y Grisar, junto a los socios gestores Arthur Vorwerk y P. J. Craseman, además de tres socios que permanecían en Alemania. ${ }^{31}$ Para 1933, al reconstituirse la firma, aparece Hans W. Vorwerk Arnold, nuevo socio comanditario que se incorpora a los anteriores. Para esa ocasión declararon como capital 350000 pesos. ${ }^{32}$ La empresa se mantuvo posteriormente, superando las adversidades de la segunda guerra mundial, cuando apareció en las listas negras elaboradas

${ }^{27}$ Jara y Muirhead, Chile, 1929, p. CLXXII.

${ }^{28}$ Ibid.

${ }^{29}$ Soto, Alemanes, 1917 , p. 56.

${ }^{30}$ Lloyd, Impresiones, 1915 , p. 382.

${ }^{31}$ Jara y Muirhead, Chile, 1929, p. CLXXII.

${ }^{32}$ Archivo del Registro del Conservador de Comercio y Bienes Raíces de Valparaíso (ARCCBRV), Registro Comercial Valparaíso, 1933, vol. 61, f. 1199. 
por los aliados. La empresa seguía operando hasta comienzos del siglo XXI con un índice de crecimiento de 2.8 por ciento. ${ }^{33}$

En 1912 comenzó a operar en Valparaíso Gildemeister y Cía., a cargo de Augusto Thiermann, quien estuvo en esa posición por muchos años dirigiendo la firma junto a Siegfried T. Gildemeister. Se dedicaban a operaciones financieras y compra de materiales para los trabajos propios de las salitreras e importaban maquinarias, hierro y cemento. Operaban como agentes para importantes fábricas alemanas y a fines de la década de 1920 adquirieron el molino California en Tomé. ${ }^{34}$ En 1929, August Thiermann era apoderado general y como socios aparecían Siegfried, Enrique y Augusto Gildemeister. ${ }^{35}$ Esta firma después se trasladó a Santiago, en donde aún está en funciones, dedicada fundamentalmente a la importación de maquinaria.

Anteriormente, Gildemeister operaba en Antofagasta, desde 1909, en donde poseía edificios para almacenes y depósitos que cubrían una superficie de 6400 metros cuadrados en donde se ubicaban maquinarias y materiales de construcción para la industria salitrera y minera en general. Bajo su dirección se construyeron las oficinas salitreras José Santos Ossa y Anibal Pinto, de la Compañía de Salitres de Antofagasta. ${ }^{36}$

\section{Actividad naviera}

La presencia e importancia naviera mercante alemana fue en ascenso desde el último cuarto del siglo XIX hasta el inicio de la primera guerra mundial. Para 1910, de acuerdo con publicaciones del Bureau Veritas, la flota mercante mundial era de 19342 buques a vapor y estaba encabezada por Gran Bretaña con 8754 unidades, seguida por Alemania con 1797 vapores; Estados Unidos con 992 y Francia con $851 .{ }^{37}$ En cuanto al tonelaje, Inglaterra tenía una capacidad de transporte de 17427798 toneladas brutas y Alemania de $3818627^{38}$

Para el caso de la comercialización con Chile es evidente que la disponibilidad de una buena flota mercante contribuía positivamente a facilitar la acción de los comerciantes alemanes en costas chilenas. La evolución que tiene la actividad naviera alemana en Chile marca también el desarrollo del comercio germano en el país. Es así como la llegada de navíos desde los estados alemanes a comienzos del siglo XIX era esporádica y reduci-

${ }^{33}$ Wiese, Rumbo, 2003, pp. 21-23.

${ }^{34}$ Lloyd, Impresiones, 1915 , p. 358.

${ }^{35}$ Jara y Muirhead, Chile, 1929, p. CCIV.

${ }^{36}$ Soto, Alemanes, 1917, p. 57.

${ }^{37}$ Anuarios, 1910, pp. 5-6.

${ }^{38}$ Ibid. 
da, coincidiendo con el incipiente comercio que se desarrollaba por parte de compañías alemanas en el país, especialmente previo a la unificación de los Estados alemanes. El Mercurio de Valparaíso mencionaba un navío hamburgués y otro bremense para 1828, tres hamburgueses para 1829, tres bremenses en 1831, y para 1836, al menos cuatro hamburgueses y dos bremenses. Los prusianos se hicieron presente con uno en 1827, 1831 y 1832 y siete en 1836. Las naves hamburguesas que aparecían eran las barcas Diana y Pilot y los bergantines Nautilus, María y Comet. Se señala, además, que varios navíos daneses venían con carga desde Hamburgo. ${ }^{39}$

Un destacado comerciante alemán, Eduard W. Berckemeyer, que operaba en distintos países de América del sur, hizo notar, en su diario, la transformación que tuvo Valparaíso en el lapso de diez años, cuando volvió al puerto en 1838. Señalaba que en su primera visita, en 1828, en un año entraban 100 navíos extranjeros y al menos 80 o 90 tenían que salir en lastre; en cambio, para 1838, ingresaban al puerto 1000 naves y, al contrario de la década anterior, los buques extranjeros llegaban a Valparaíso en lastre para cargar productos chilenos. ${ }^{40}$

Para 1857, llama la atención la aparición de ocho alemanes propietarios de buques, cuya actividad se vinculaba estrechamente con el comercio mayorista. ${ }^{41}$

Lograda la unificación alemana se constituyó un tráfico naviero más estable, especialmente después de que el 28 de noviembre de 1872 se fundara la naviera Deutsche Dampfschiffahrts-Gesellschaft Kosmos como iniciativa de un grupo de armadores y corredores de Hamburgo. Kosmos inició sus viajes cada mes con seis barcos, que tenían nombres procedentes de la mitología griega. La ruta que realizaban partía desde Hamburgo, vía Le Havre, para atravesar Montevideo y continuar hacia Buenos Aires, Valparaíso, Arica y El Callao. Ante el éxito de la empresa pronto se amplió la flota, zarpando cada quince días. Se trataba fundamentalmente de buques cargueros con reducidas capacidades para pasajeros. ${ }^{42}$

En 1886 Adolph Kirsten, también de Hamburgo, creó la naviera Hamburg Pacific Dampfschiffslinie para operar en la misma ruta de la Kosmos. Se inició una competencia que provocó la disminución de las tarifa de los fletes. En 1894 ambas compañías lograron un acuerdo creando un pool

${ }^{39}$ Kellenbenz, "Eduard”, 1987, pp. 27-29.

${ }^{40}$ Ibid., pp. 39-40.

${ }^{41}$ Entre los dueños de buques, dos de ellos eran propietarios de dos naves, H. Dam y Guillermo Lutjen. Los otros propietarios eran J. F. Garbe, F. Schonberg, J. Handt, B. Dietjten, J. Bunssanth y Hans C. Petersen. Véase Guí, 1858.

${ }^{42}$ Wiese, Rumbo, 2003, p. 57. 
en forma conjunta. Posteriormente, en 1898, Kosmos se hizo cargo de la participación de Hamburg Pacific Linie. ${ }^{43}$

A comienzos del siglo Xx Kosmos tenía 28 grandes vapores y mantenía siete salidas mensuales desde Hamburgo a las costas del Pacífico. En Chile se concentraban fundamentalmente en el transporte de salitre. ${ }^{44}$ La nueva flota de vapores también poseía muy buenas instalaciones para pasajeros. Complementaba la labor de las diversas compañías navieras que abastecían el mercado chileno una empresa que se estableció en 1909 bajo la denominación de Compañía Marítima Alemana y que se dedicó a apoyar los trabajos portuarios con flete de lanchas, descargas, embarques, remolques, proporción de lastres, etc. Su capital en 1920 era de 400000 pesos. ${ }^{45}$ Ambas navieras estaban representadas en Chile por Vorwerk.

De acuerdo con el cuadro 4 podemos advertir la evolución que tiene la actividad mercantil alemana, específicamente en su relación con Valparaíso, mostrando una trayectoria ascendente desde mediados del siglo XIX hasta inicios del XX, para luego marcar un descenso notorio en el transcurso posterior que afectó también a Gran Bretaña. Entre las causales de tal deterioro naviero y mercantil surgen varias, tanto de origen interno como de procedencia externa. La actividad mercantil internacional, a partir de 1880, comenzó a verse afectada por una notoria disminución en su movimiento. Por una parte era la manifestación del término de la importancia del ciclo minero del cobre, que estuvo concentrado en el manejo mercantil y financiero desde Valparaíso, a lo que se sumó la mencionada crisis económica que afectó al país en la década de 1870. La etapa posterior, a cargo del desarrollo salitrero, cambió el eje económico del país desde Valparaíso hacia el norte.

A lo anterior, cabe señalar, a comienzos del siglo xx, la evolución de la infraestructura comunicacional continental, como fue el caso del desarrollo del ferrocarril de San Francisco a Nueva York y el ferrocarril a través del istmo de Panamá. Estos factores, además del inicio de las operaciones del canal de Panamá, fueron importantes en la pérdida de importancia del puerto de Valparaíso. ${ }^{46}$

Por otro lado, cabe consignar también la transición desde la navegación a vela a la de vapor, que dio mayor autonomía a los buques haciendo innecesario el recalamiento en Valparaíso para muchas embarcaciones que llegaban al Pacífico vía el estrecho de Magallanes. Este mismo hecho debe considerarse también en cuanto a la ponderación de la presencia

${ }^{43}$ Ibid., p. 59.

${ }^{44}$ Lloyd, Impresiones, 1915, p. 381.

${ }^{45}$ Aranda, Llarena y Tenajo, Colonia, 1920, p. 186.

${ }^{46}$ Fagalde, Puerto, 1903, p. 80. 
CUADRO 4. INGRESO DE NAVES EUROPEAS

A VALPARAÍSO, 1851-1931

\begin{tabular}{lccccccr}
\hline \multicolumn{1}{c}{ Gran } & & & & & & \\
Años & Bretaña & Alemania & Francia & Italia & España & Otros & Total \\
& & & & & & & \\
1851 & 252 & 58 & 37 & 7 & 2 & 470 & 826 \\
1861 & 245 & 44 & 51 & 4 & 12 & 650 & 1006 \\
1881 & 386 & 94 & 38 & 22 & s. d. & 83 & 623 \\
1890 & 306 & 132 & 50 & 2 & 1 & 104 & 595 \\
1901 & 321 & 125 & 7 & 15 & s. d. & 100 & 568 \\
1910 & 310 & 178 & 16 & 13 & 2 & 102 & 621 \\
1921 & 80 & 24 & 9 & 11 & s.d. & 254 & 378 \\
1931 & 43 & 75 & 18 & s. d. & s.d. & 143 & 279 \\
\hline
\end{tabular}

Fuentes: Estadística, 1851-1901, y Anuarios, 1910-1931.

cuantitativa de naves, por cuanto el incremento de buques a vapor determinó asimismo un aumento en la capacidad de carga de cada unidad de transporte. Para 1880 ingresaron a Valparaíso 714 barcos, de los cuales 189 eran vapores y 525 veleros, con un promedio de carga de 823 toneladas por barco. Para 1900 el ingreso fue de 798 barcos, donde 623 eran vapores y 175 veleros, con un promedio de 1867 toneladas por navío. ${ }^{47}$

\section{Sistema bancario}

La colectividad disponía de un eficiente sistema bancario que agilizaba y apoyaba sus actividades financieras. A fines del siglo XIX aparecieron casi en forma simultánea dos instituciones generadas desde Alemania. En 1895 se instaló en Valparaíso el Banco de Chile y Alemania, fundado por la Direction der Disconto Gesellschaft de Berlín, y el Norddeutsche Bank Hamburg. Para 1925 los gerentes en Valparaíso eran Gust L. Berckemeyer y Emilio Mundigo. Había también sucursales en Santiago, Antofagasta, Concepción, Valdivia y Temuco, y el capital declarado para Chile era de 10000000 de pesos. ${ }^{48}$

Otra institución financiera alemana establecida en Valparaíso fue el Banco Alemán Transatlántico, que se instaló en Chile en 1896, dependien-

${ }^{47}$ Ibid., p. 115.

${ }^{48}$ Pellegrini y Aprile, Progreso, 1924, p. 268. 
te del Deutsche Ueberseeischen Bank de Berlín. Poseía sucursales en Santiago, Concepción, Temuco, Valdivia, Antofagasta e Iquique. En Valparaíso los directores eran K. Hüttman y H. Kratzer. ${ }^{49}$ A fines de 1909 llegó a Valparaíso Paul Wallich, hijo de uno de los miembros del directorio del Detschen Überseeischen Bank, en visita como veedor de todos los bancos en el continente americano.

En opinión de Wallich, luego del Banco de Chile, que llevaba las cuentas del Estado chileno, venía en importancia el Banco Alemán Transatlántico y luego el Banco Español de Chile. ${ }^{50}$ De acuerdo con el balance de 1910, el Banco de Chile poseía un capital de 320610812 pesos, el Banco Español de Chile 121355376 pesos y el Alemán Transatlántico 120568416 pesos. ${ }^{51}$ Para 1910 los tres bancos alemanes representaban $22.18 \%$ del capital bancario que reunían la totalidad de los bancos establecidos en Valparaíso, que disminuyó a 9.8\% en 1920, luego de terminada la primera guerra mundial (1918). ${ }^{52}$ Evidentemente, esta disminución tuvo que ver con los resultados de la guerra y los consecuentes efectos que esta tuvo para el comercio internacional alemán.

Una tercera institución bancaria fue el Banco Germánico de la América del Sur, que se estableció en Chile en 1910 como sucursal del Deutsch Suedamerickanische Bank. También se estableció en Santiago. ${ }^{53}$

\section{Compañias de seguros}

En general, este tipo de organizaciones estaban destinadas a otorgar seguros de toda clase de riesgos, contra incendios, lucro cesante, marítimos y de transportes terrestres. Se vinculaban estrechamente con las casas consignatarias o importadoras, las que operaban como agencias. Al igual que las casas importadoras, se percibe bastante movilidad en el caso de las compañías aseguradoras. Es así como las compañías existentes a comienzos del siglo XX desaparecen posteriormente, y son reemplazadas por otras. Al igual que lo que ocurrió en general en toda la actividad económica, la guerra fue evidentemente la principal causal de estos cambios.

Estas compañías desempeñaban una labor importante para las compañías navieras y para los importadores, ya que procuraban mantener una mayor seguridad en sus inversiones. De allí su gran permanencia como expresión de la relevancia mercantil alemana (véase cuadro 5).

${ }^{49}$ Ibid., p. 264.

${ }^{50}$ Wallich, Banco, 1986, p. 141.

${ }^{51}$ Anuarios, 1910, pp. 608-609.

${ }^{52}$ Anuarios, 1910 y 1920.

${ }^{53}$ Pellegrini y Aprile, Progreso, 1924, p. 270. 


\section{CUADRO 5. COMPAÑIIAS ALEMANAS DE SEGUROS CON AGENCIAS EN VALPARAÍSO, 1903}

Nombre

Aachen y Munchener de Aachen

La Hamburgo Bremense

Imperial Insurance Co. Ltd.

Law Union and Crown Insurance Co.

La Magdeburg

Nord Deutsche Insurance Co.

La Transatlántica

National Prusiana de Stettin
Agente

Weber y Cía.

J. E. Rücker

Chodowiecki y Cía.

James A. Moir

Robert Maas

Eberhard Focke

D. F. C. Walter

E. Lütjens

Fuente: Fagalde, Puerto, 1903.

Entre las compañías de seguros que estaban controladas por la colectividad, hacia 1925, tenemos la Compañía de Seguros Germania, que poseía en 1920 un capital suscrito de 2000000 de pesos y un directorio compuesto por J. E. Pini (presidente), Walter Bade (vicepresidente), Karl Hüttmann, Carlos Menke, S. T. Gildemeister, Otto Hildebrandt, B. Timmerman y N. Lafrenz. ${ }^{54}$ Cinco años más tarde el directorio estuvo compuesto por Walter Bade (presidente), Jorge Chodowiecki, S. T. Gildemeister, Otto Hildebrandt, Carlos Menke, H. Kratzer, G. Friederichs y N. Lafrenz (secretario gerente) ${ }^{55}$ La compañía de seguros Araucanía se vinculaba a la Germania y poseía su mismo directorio, capital y local operacional. Su secretario gerente, en 1925, era Oscar Bordalí.

En 1920 apareció la compañía de seguros La Transandina, que luego se vinculó con la Caupolicán cuando esta apareció en 1925. La Transandina poseía, en 1929, un capital de 2647507 pesos y su director gerente era Erich Wichmann y el subgerente Germán Glimmann. Por su parte, la Caupolicán, en igual fecha, tenía un capital de 1628321 pesos y su gerente era también Germán Glimmann. Ambas poseían sucursales en todo el país. ${ }^{56}$ Una quinta compañía de seguros controlada por miembros de la colectividad alemana fue La Confianza, que operaba contra incendios y riesgos marítimos y para 1925 tenía un capital de 1600000 pesos. Su

\footnotetext{
${ }^{54}$ Aranda, Llarena y Tenajo, Colonia, 1920, p. 172.

${ }_{55}^{55}$ Pellegrini y Aprile, Progreso, 1924, p. 272.

${ }^{56}$ Jara y Muirhead, Chile, 1929, p. 123.
} 
directorio estaba constituido por Roberto Maass (presidente), Edgar Telge (vicepresidente), Adolfo Wilkens, Teodor Kunze, August Thiermann, Otto Framm y Fernando Stimmann como director-gerente. ${ }^{57}$

$\mathrm{Al}$ observar los nombres de los directores de las diversas compañías aseguradoras se advierte la coincidencia con altos ejecutivos de casas importadoras como de bancos alemanes establecidos en la ciudad. Por otro lado, se advierte que luego del conflicto bélico de 1914 se reconstituyó la estructura de estas instituciones, las cuales, en todo caso, mantuvieron esa íntima relación que siempre tuvieron con las casas importadoras.

\section{Término del ciclo}

La trayectoria económica de Chile desde mediados del siglo XIX está marcada por la fuerte dependencia que tenía de la exportación de productos primarios. Inicialmente fue la minería de la plata y cobre para, luego del triunfo en la guerra del Pacífico, transferir la primacía tributaria a la exportación del salitre. Esta evolución determinó, en parte, la disminución de la importancia de Valparaíso como centro económico-financiero a fines del siglo XIX. En 1851 salía de Valparaíso 73\% de las exportaciones del país y se importaba $58 \%$; para 1882 las importaciones equivalían a $79 \%$ y las exportaciones 19\% del total nacional. Para 1915 la situación cambió radicalmente: sólo se exportaba 5\% y se importaba 49\% del total nacional. ${ }^{58}$

El funcionamiento del puerto de San Antonio en 1918, más cercano a Santiago, se sumó a los otros factores, ya señalados, que afectaron la actividad portuaria de Valparaíso, como consecuencia de la modernización y ampliación de los medios de transporte y comunicaciones.

Por otro lado, para 1915 la situación comercial de los alemanes en Valparaíso, como consecuencia del estallido de la guerra mundial, experimentó un proceso de decadencia que afectó de manera sustantiva sus intereses. Tal como lo muestran los cuadros 6 y 7 , la presencia alemana en el comercio chileno cae de manera sustantiva. Previo al estallido de la guerra, los vínculos anglo-germanos eran comunes, por lo cual el conflicto determinó rupturas que afectaron significativamente el quehacer comercial de ambos grupos, llevando la peor parte los alemanes. Las relaciones entre ambos colectivos europeos, previo al conflicto, eran de tal cercanía que el vicecónsul británico de Caleta Coloso era un súbdito alemán, quien fue cambiado de inmediato, luego de declarada la guerra ${ }^{59}$ En Valparaíso,

\footnotetext{
${ }^{57}$ Aranda, Llarena y Tenajo, Colonia, 1920, p. 278.

${ }^{58}$ Schmutzer, "Puerto", 2000, p. 106.

${ }^{59}$ Couyoumdjiam, Chile, 1986, p. 59.
} 
Am. Lat. Hist. Econ., año 20, núm. 2, mayo-agosto, 2013, pp. 151-176

CUADRO 6. IMPORTACIONES POR VALPARAÍSO

SEGÚN NACIÓN DE ORIGEN, 1882-1926 (NAVES/TONELADAS)

\begin{tabular}{ccccccc}
\hline Años & Gran Bretañ & Alemania & Francia & EUA & Chile & Otros \\
& & & & & & \\
1882 & 60.3 & 12.3 & 7.8 & 5.7 & 8.2 & 5.1 \\
1890 & 51.5 & 22.6 & 9.3 & 4.8 & 9.3 & 2.6 \\
1912 & 45.6 & 35.2 & 3.5 & 2.0 & 8.6 & 5.0 \\
1919 & 33.0 & s. d. & 1.1 & 23.7 & 26.0 & 16.0 \\
1926 & 29.6 & 17.1 & 5.0 & 11.3 & 16.2 & 21.1 \\
\hline
\end{tabular}

Fuente: Schmutzer e Ibáñez, "Valparaíso", 1986, p. 156.

\section{CUADRO 7. IMPORTACIONES EUROPEAS A CHILE SEGÚN NACIÓN DE ORIGEN, 1850-1930 (EN PORCENTAJES)}

\begin{tabular}{lcrrrrr}
\hline Años & Gran Bretaña & Alemania & Francia & Italia & España & Suma \\
& & & & & & \\
1850 & 35.36 & 8.28 & 11.39 & 0.50 & 0.97 & 56.50 \\
1860 & 34.04 & 7.57 & 21.68 & 0.35 & 2.88 & 66.52 \\
1870 & 45.20 & 9.52 & 21.45 & 0.76 & 0.45 & 77.38 \\
1880 & 45.08 & 16.10 & 14.80 & 0.99 & 2.02 & 78.99 \\
1890 & 43.42 & 23.09 & 10.08 & 0.65 & 0.39 & 77.63 \\
1900 & 33.05 & 26.70 & 7.22 & 1.73 & 0.62 & 69.32 \\
1910 & 31.62 & 24.21 & 6.45 & 2.97 & 1.15 & 66.40 \\
1920 & 25.52 & 4.63 & 6.21 & 1.24 & 2.01 & 39.61 \\
1930 & 15.26 & 16.89 & 5.01 & 3.26 & 1.22 & 41.64 \\
\hline
\end{tabular}

Fuentes: elaboración propia con base en Estadística, 1882, 1890-1891, 1900, y Anuarios, 1910, 1920 y 1930.

el Banco Anglo Sud Americano tenía un gerente alemán, de apellido Greve, quien fue relevado de sus funciones. ${ }^{60}$

El cónsul británico Maclean, en Valparaíso, lideró una campaña para conseguir que todas las agencias que estuvieran en manos de alemanes fueran reemplazadas. Por su parte la Pacific Steam Navigation Company,

${ }^{60}$ Ibid., p. 60. 
de propiedad británica, se negó a transportar súbditos enemigos en sus servicios regulares entre puertos chilenos, violando la neutralidad de Chile. ${ }^{61}$

Entre las actividades más importantes que propició el cónsul Maclean están la creación de la Cámara de Comercio Británica en Chile, en 1917, y la elaboración de las denominadas "listas negras", en donde se señalaban las casas comerciales (alemanas o con vínculos alemanes) o personas con quienes no se debía tener ningún tipo de relaciones comerciales. ${ }^{62}$ Esta lista llegó a consignar 281 empresas o comerciantes hasta abril de 1919. De esta cifra, 87 estaban establecidos en Valparaíso. ${ }^{63}$

Esta disminución en la participación económica de Alemania en el comercio chileno se expresó también en la estructura demográfica de la colectividad establecida en Valparaíso. La mayor cifra que alcanza el grupo se produce con el censo de 1907 (véase cuadro 1), que es también el periodo cuando se logra el mayor porcentaje de incidencia del comercio alemán en el país (véanse cuadros 6 y 7). Posteriormente viene un descenso tanto en la presencia demográfica como en la comercial.

\section{CONCLUSIONES}

Al tenor de la información precedente es perceptible la relevancia que tuvieron las casas consignatarias o importadoras en la actividad económica llevada a cabo en Valparaíso en relación con el comercio internacional. Efectivamente, existía una estructura instalada que vinculaba los diversos ámbitos del comercio internacional que facilitaban las operaciones efectuadas en el primer puerto de Chile. Es así como la existencia de bancos, compañías de seguros, compañías navieras y casas comerciales conformaba un conjunto cohesionado de servicios íntimamente relacionados, que facilitaban el quehacer comercial alemán en Chile y también en el continente americano, por cuanto Valparaíso era sólo un eslabón de una extensa y potente cadena económica dentro del Pacífico. En esta cuádruple convergencia institucional es palmaria la labor de eje principal que tenían las casas importadoras, las cuales en sus orígenes también operaban como bancos y tenían a su cargo la representación de las compañías aseguradoras, de las compañías navieras y la atención de naves.

Las características que tuvo la actividad económica de Valparaíso favorecieron de modo significativo la actividad comercial de los alemanes. $\mathrm{Al}$ mismo tiempo, la estructura cimentada por estas empresas facilitó las

\footnotetext{
${ }^{61}$ Ibid., p. 61.

${ }^{62}$ Ibid., p. 146.

${ }^{63}$ Ibid., pp. 291-328.
} 
operaciones de otros comerciantes y profesionales que se instalaron como comerciantes o empresarios privados dentro del comercio urbano o prestando sus servicios profesionales. De esta manera encontramos también, junto a la presencia de las casas mayoristas, establecimientos como farmacias, cervecerías, casas de venta de artículos musicales, etc., conjuntamente con arquitectos e ingenieros que disfrutaron de una amplia acogida en el medio local.

La comprensión del fenómeno comercial del puerto de Valparaíso y la participación de los colectivos inmigrantes europeos se insertan en el proceso de globalización vivido a fines del siglo XIX. Es así como se advierte una relación directa en cuanto a la relevancia que tienen los respectivos colectivos en la actividad económica local y su posicionamiento en el comercio internacional. De allí que, en el caso de los alemanes, se perciba una ingente actividad económica en Valparaíso potenciada y apoyada por la infraestructura económico-financiera que operaba en la globalizada economía liderada por Gran Bretaña y escoltada por Alemania.

\section{Fuentes CONSULTADAS}

\section{Archivos}

ARCCBRV Archivo del Registro del Conservador de Comercio y Bienes Raíces de Valparaíso.

\section{Hemerografia}

El Heraldo, Valparaíso, Chile.

El Mercurio, Valparaíso, Chile.

La Patria, Valparaíso, Chile.

\section{Bibliografía}

Anuarios estadísticos de la República de Chile, Chile, Oficina Central de Estadística de Chile, años 1910-1931.

aranda Diego, José María Llarena y Rafael Tenajo, La colonia alemana en Chile, Santiago de Chile, Claret, 1920, 835 pp.

Blancpain, Jean Pierre, Les allemands au Chili: 1816-1943, Colonia, Böhlau Verlag, 1974, 1162 pp. 
Cavieres, Eduardo, Comercio chileno y comerciantes ingleses, 1820-1880: un ciclo de historia económica, Valparaíso, Universidad Católica de Valparaíso, 1988 (Serie Monografías Históricas núm. 2).

Censos generales de la República de Chile, años 1865, 1875, 1885, 1895, 1907, 1920 y 1930. COUyOumdjiAm, J. RicARDO, Chile y Gran Bretaña durante la primera guerra mundial y la postguerra, 1914-1921, Santiago, Andrés Bello/Universidad Católica de Chile, 1986.

, "El alto comercio de Valparaíso y las grandes casas extranjeras, 18801930. Una aproximación”, Historia (Santiago), Pontificia Universidad Católica de Chile, vol. 33, 2000, Santiago de Chile, pp. 63-99.

Chaigneau, Julio (ed.), Guía comercial de Valparaíso 1874-1875, Valparaíso, Imp. La Patria, 1874.

Douglas MAsSEY et al., "Teorías sobre la migración internacional: una reseña y una evaluación", Population and Development Reviere, Population Council, vol. 19, núm. 3, septiembre de 1993, Nueva York, pp. 431-466.

Estadística Comercial de la República de Chile, Chile, Departamento de la Estadística Comercial, República de Chile, años 1882, 1851-1901.

FAgAlde, Alberto, El puerto de Valparaíso y sus obras de mejoramiento, Holanda, Tipografía Van Marken, 1903.

Garreau, Jacqueline, "La formación de un mercado de tránsito. Valparaíso: 18171848”, Nueva Historia, Asociación de Historiadores Chilenos, vol. 3, núm. 11, 1984, Londres, pp. 157-194.

Graham, María, Diario de mi residencia en Chile, Santiago de Chile, Francisco de Aguirre, 1988.

Guía de Valparaíso y Santiago o repertorio general para el año 1857, Valparaíso, Imprenta del Comercio, 1858.

"Guía general de Chile 1847" en José ViCTORINo LASTARria, Lecciones de geografía moderna, Valparaíso, Imprenta el Mercurio, 1846.

Guía universo. Índice general de la república de Chile, 1925-1926, Santiago, Sociedad Imprenta y Litografía Universo, 1926.

Herrera Balharry, Eugenio, Los alemanes y el Estado cafetalero, San José de Costa Rica, Universidad Estatal a Distancia, 1988.

Jara Letelier, Anibal y Manuel G. Muirhead, Chile en Sevilla (El progreso material, cultural e institucional de Chile en 1929), Santiago de Chile, Empresa Editorial Cronos, 1929.

Kellenbenz, Hermann, "Eduard Wilhelm Berckemeyer, mercader hamburgués en Valparaíso (1837-1838)”, Historia, Pontificia Universidad Católica de Chile, núm. 22, 1987, Chile.

, "Relaciones consulares entre las ciudades hanseáticas y Chile: el caso de Valparaíso hasta los años 1850", Jahrbuch fur Geschichte von Staat, Wirtschaft und Gesellschaft, Böhlau, núm. 25, 1988, Koln, pp. 117-140. 
y JÜRGEn SchneIder, "La emigración alemana a América Latina desde 1821 hasta 1930", Jahrbuch fur Geschichte von Staat, Wirtschaft und Gesellschaft, Böhlau, núm. 13, 1976, Koln, pp. 386-403.

Lloyd, Reginald, Impresiones de la república de Chile en el siglo XX, Londres, Jus Truscott and Son Ltd., 1915.

Mentz, Brígida von, Los pioneros del imperialismo alemán en México, México, Centro de Investigaciones y Estudios Superiores en Antropología Social/Ediciones de la Casa Chata, 1982, vol. 14.

Murray, Andrew, Home from the Hill. A Biography of F. Huth, Napoleon of the City, Londres, Hamilton, 1970, 242 pp.

Ortega Martínez, Luis, Chile en ruta al capitalismo: cambio, euforia y depresión 18501880, Santiago de Chile, Dirección de Bibliotecas, Archivos y Museos/Ediciones LOM Ediciones, 2005.

PARodi, CARlos, "América Latina y la globalización económica: una visión de largo plazo", Lima, Centro de Investigación de la Universidad del Pacífico, 2007 (Documento de Discusión, núm. DD/07/02).

Pellegrini y Aprile (eds.), El progreso alemán en Chile, t. I: Chile 1924, Santiago de Chile, Editorial Río de la Plata, 1924.

Pena de la Fuente, Nicolás, Guía general de Valparaíso y Viña del Mar, Santiago, Imprenta Barcelona, 1913.

SCHMUTZER, KARIN y AdOLFO IbÁÑEZ, "Valparaíso como lugar de encuentro entre Chile y el mundo" en AA. VV., Valparaíso, 1536-1986, Viña del Mar, Universidad Católica de Valparaíso/Altazor, 1986 (Serie Monografías Históricas, núm. 1).

SCHMUTZER, KARIN, "El puerto: comercio, ingreso, ingresos, los hombres e infraestructura” en BALDOMERO ESTRADA et al., Valparaíso, sociedad y economía en el siglo XIX, Valparaíso, Universidad Católica de Valparaíso/Ediciones Universitarias de Valparaíso, 2000 (Serie Monografías Históricas, núm. 12).

SEYFerth, Giralda, "Inmigración alemana y la política brasileña de colonización”, Estudios Migratorios Latinoamericanos, Centro de Estudios Migratorios Latinoamericanos, vol. 10, núm. 29, 1995, Argentina, pp. 53-75.

Solé, Carlota, Sonia Parella y Leonardo Calvancanti, El empresariado inmigrante en España, Barcelona, Fundación La Caixa, 2007.

Soto Rojas, SAlvador, Los alemanes en Chile 1541-1917. Progresos y servicios que les debe la república, Valparaíso, Imprenta Victoria, 1917.

Vera de Flachs, MARÍA CRISTina, "Emigraciones transoceánicas. Los alemanes en América. 1850-1914. El caso argentino”, Cuadernos de Historia Contemporánea, Universidad Complutense de Madrid, núm. 16, 1994, Madrid, pp. 65-98.

Vicuña Mackenna, Benjamín, Bases del informe presentado al supremo gobierno sobre la inmigración extranjera, Santiago de Chile, Imprenta Nacional, 1865.

Viebrock, Hans Heinrich, "Historia del Colegio Alemán 1857-1997” en Colegio Alemán de Valparaíso, 140 Años, Edición Aniversario, Valparaíso, Litografía Carroza, 1997. 
VILlarino, JoAQUín, Estudios sobre la colonización y emigración europea a Chile, Santiago de Chile, Imprenta Nacional, 1867.

Wallich, Paul, Banco Alemán Transatlántico, Mainz, Hase \& Koehler, 1986.

Wiese, Eigel, Rumbo a Chile 1822-2003. Testimonios de relaciones navieras chileno-alemanas, Santiago de Chile, Morgan Impresores, 2003.

Young, George F. W., Germans in Chile: Immigration and Colonization, 1849-1914, Nueva York, Center for Migration Studies, 1974. 